\title{
Influence of alkyl chain substitution on sexithienyl-metal interface morphology and energetics
}

\author{
S. Duhm, H. Glowatzki, J. P. Rabe, and N. Koch ${ }^{\text {a) }}$ \\ Institut für Physik, Humboldt-Universität zu Berlin, Newtonstrasse 15, D-12489 Berlin, Germany \\ R. L. Johnson \\ Institut für Experimentalphysik, Universität Hamburg, D-22761 Hamburg, Germany
}

(Received 3 January 2006; accepted 31 March 2006; published online 16 May 2006)

\begin{abstract}
The interface between $\mathrm{Ag}(111)$ and vacuum sublimated $\alpha, \omega$-dihexylsexithienyl (DH6T) was investigated using ultraviolet photoelectron spectroscopy and atomic force microscopy. While the monolayer of DH6T is lying flat on the metal surface, we found that already in the second molecular layer the molecules are almost standing upright. This abrupt change in molecular orientation lowered the hole injection barrier $\left(\Delta_{h}\right)$ of DH6T/Ag by $0.5 \mathrm{eV}$ between monolayer and multilayer. $\Delta_{h}$ for DH6T multilayers was even lowered by $0.8 \mathrm{eV}$ compared to unsubstituted sexithienyl multilayers. The reduction of $\Delta_{h}$ is attributed to the electronic decoupling of molecules in the first from those in the second layer via the hexyl chains. (C) 2006 American Institute of Physics.
\end{abstract}

[DOI: 10.1063/1.2204834]

The energy level alignment at metal/organic interfaces is a key issue for improved performance of organic (opto-) electronic devices. ${ }^{1-3}$ In order to enhance charge carrier injection and the efficiency of devices, such as organic light emitting diodes, solar cells, or field effect transistors, lowering of the hole injection barrier is indispensable. ${ }^{4}$ Poly- and oligothienylenes are widely used in organic electronic devices, which exhibit excellent performance., 5 Regarding industrial-scale device fabrication, solution-based processing of organic materials is often preferred over vacuum sublimation. Therefore, alkyl chains are frequently attached to the electroactive molecular backbone, rendering these materials highly soluble.

One may not expect significant changes in the organic/ metal interface electronic properties due to simple addition of alkyl chains (compared to the unsubstituted oligomer) because of the insulating nature of the saturated alkyls. In fact, $\beta \beta^{\prime}$-dihexylsexithienyl exhibited essentially the same energetics at interfaces to $\mathrm{Au}$ as $\alpha$-sexithienyl (6T). ${ }^{7}$ However, the growth of molecules may be substantially changed by alkyl chain addition. ${ }^{8,9}$ Consequently, the electronic structure of a molecular film and also its interface electronic properties may depend on different molecular organization. ${ }^{10}$

For the case of the 6T derivative $\alpha, \omega$ - dihexylsexithienyl [DH6T; chemical structure shown in Fig. 1(a)] on $\mathrm{Ag}(111)$ we found a significant change in growth mode compared to the unsubstituted $6 \mathrm{~T},{ }^{11,12}$ which, in conjunction with the electrically insulating behavior of the hexyl chains, led to a dramatic reduction of the hole injection barrier towards a metal electrode.

Ultraviolet photoelectron spectroscopy (UPS) experiments were carried out at the FLIPPER II end-station at HASYLAB (Hamburg, Germany). ${ }^{13}$ The interconnected sample preparation chambers (base pressure $2 \times 10^{-9} \mathrm{mbar}$ ) and analysis chamber (base pressure $2 \times 10^{-10}$ mbar) allowed sample transfer without breaking ultrahigh vacuum (UHV) conditions. The $\mathrm{Ag}(111)$ single crystal was cleaned by re-

\footnotetext{
${ }^{\text {a) }}$ Author to whom correspondence should be addressed; electronic mail:
} norbert.koch@physik.hu-berlin.de peated Ar-ion sputtering and annealing cycles (up to $550{ }^{\circ} \mathrm{C}$ ) until a clear low energy electron diffraction pattern was observed. DH6T (H. C. Starck GmbH, Product No. BSX 1728/1) and 6T (Aldrich, Product No. 594687) were evaporated using resistively heated pinhole sources, at evaporation rates of about $1 \AA /$ min. The film mass thickness was monitored with a quartz crystal microbalance. Spectra were recorded with a double-pass cylindrical mirror analyzer with an energy resolution of $0.15 \mathrm{eV}$ (measured as $80 \%$ to $20 \%$ intensity drop for a metal Fermi edge) and a photon energy of $22 \mathrm{eV}$. The secondary electron cutoffs (CO) (for determination of the sample work function) were measured with the sample biased at $-3.00 \mathrm{~V}$. To avoid sample charging during photoemission from multilayer DH6T films, spectra were recorded with an Al foil in the photon beam, reducing the photon flux by a factor of seven.

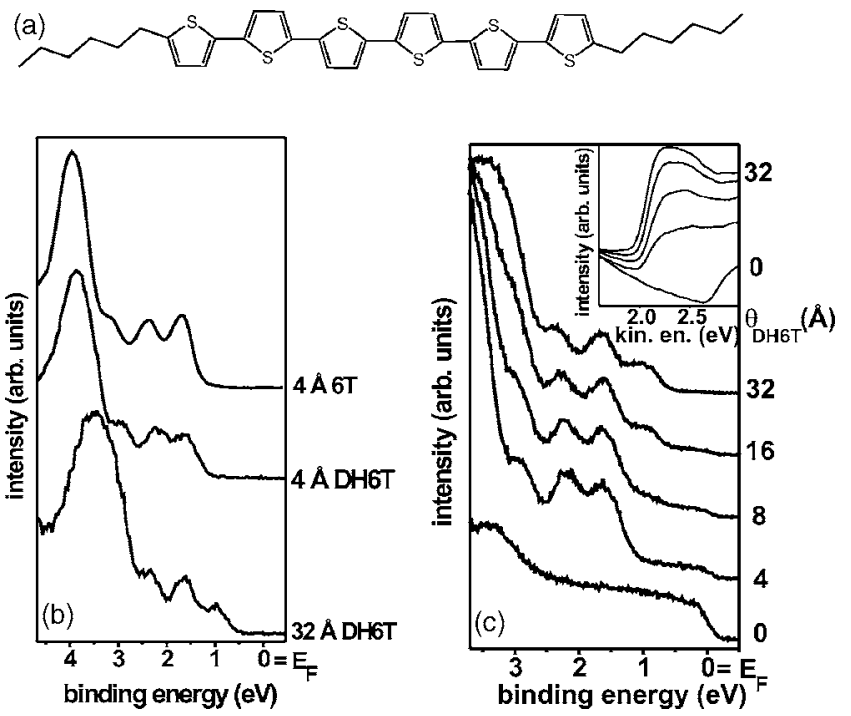

FIG. 1. (a) Chemical structure of dihexylsexithienyl (DH6T). (b) UPS spectra of $4 \AA 6 \mathrm{~T} / \mathrm{Ag}$ (111) (top), $4 \AA$ DH6T/Ag(111) (middle), and $32 \AA \mathrm{DH} 6 \mathrm{~T} /$ $\operatorname{Ag}(111)$ (bottom). The contribution from the Ag substrate was subtracted from both $4 \AA$ films (Ref. 14). (c) Low BE range photoemission spectra for increasing DH6T coverage $\left(\theta_{\mathrm{DH} 6 \mathrm{~T}}\right)$ on $\mathrm{Ag}(111)$. The inset shows the secondary electron cutoff; "kin. en." means kinetic energy. 


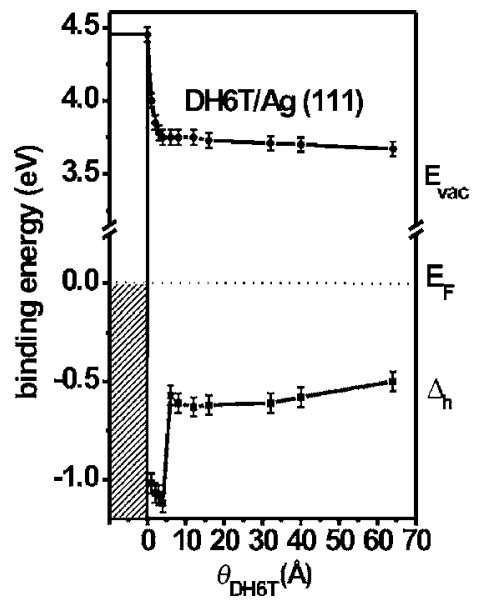

FIG. 2. Schematic energy level diagram (derived from UPS) for DH6T on $\operatorname{Ag}(111) . \theta_{\mathrm{DH} 6 \mathrm{~T}}$ denotes the DH6T thickness, $E_{\mathrm{vac}}$ the vacuum level, and $\Delta_{h}$ the hole injection barrier (HOMO onset). $E_{F}$ is the Fermi level of the metal substrate.

Atomic force microscopy (AFM) experiments were performed at Humboldt-Universität in a custom UHV system (base pressures of $1 \times 10^{-9}$ and $1 \times 10^{-10} \mathrm{mbar}$ in the preparation and analysis chambers, respectively) equipped with an Omicron VT-AFM, operated in noncontact mode. Sample preparation was analogous to that for UPS measurements.

The overall shape of the photoemission spectra of ca. a monolayer (nominal $4 \AA$ ) of $6 \mathrm{~T}$ and DH6T on $\mathrm{Ag}(111)$ is expectedly very similar [see Fig. 1(b)], since the conjugated moieties of both molecules are the same. However, the DH6T spectrum is rigidly shifted towards lower binding energy (BE). Close inspection reveals that the hole injection barrier $\Delta_{h}$ [defined as the energy difference between the low $\mathrm{BE}$ onset of the highest occupied molecular orbital (HOMO) and the metal Fermi level] is $0.15 \mathrm{eV}$ smaller for a monolayer of DH6T compared to a monolayer of $6 \mathrm{~T}^{14}$ The same energy difference is found for the ionization energies (IEs) of the two thin film samples $\left(\mathrm{IE}_{6 \mathrm{~T}}=5.0 \mathrm{eV}\right.$ and $\mathrm{IE}_{\mathrm{DH} 6 \mathrm{~T}}$ $=4.85 \mathrm{eV}$ ). The lower observed value for both $\Delta_{h}$ and IE of DH6T can be explained by increased intramolecular screening due to the additionally available electron density on the hexyl chains, which are absent for $6 \mathrm{~T}$. The similarity of $6 \mathrm{~T}$ and DH6T photoemission spectra and electronic properties suggests that in a monolayer (ML) DH6T molecules are lying flat on $\mathrm{Ag}(111)$, as would be expected on general grounds for conjugated organic molecules on that metal surface. ${ }^{11,15}$

In contrast to other organic rodlike molecules, such as pentacene, sexiphenyl, or 6 $\mathrm{T}^{2,14,16}$ the photoemission spectra of multilayer DH6T films on $\mathrm{Ag}(111)$ [bottom curve in Fig. 1(b)] are rigidly shifted towards lower BE compared to the ML. In order to investigate the origin of that unusual shift, we recorded coverage-dependent photoemission spectra of DH6T on $\mathrm{Ag}(111)$ [Fig. 1(c)]. Notably, there is no gradual shift of DH6T features, but a new emission feature appears at the low BE side of the ML HOMO (centered at ca. $0.9 \mathrm{eV}$ $\mathrm{BE}$ ) and remains at constant $\mathrm{BE}$ for increasing coverage. As evident from a comparison of Figs. 1(b) and 1(c), the HOMO of ML-DH6T coincidentally appears at the same BE as the HOMO-1 of multilayer DH6T. The new HOMO position for multilayer DH6T leads to a lowering of $\Delta_{h}$ by $0.5 \mathrm{eV}$ compared to the ML. Noteworthy, we did not observe any further change in the sample work function $(\phi)$ after the initial $\phi$ reduction due to ML formation [see inset of Fig. 1(c)]. The evolution of the interface electronic properties of DH6T on $\mathrm{Ag}(111)$ as derived from UPS is summarized in Fig. 2.

In general, $\Delta_{h}$ for organic materials deposited on metal substrates increases slightly (a few tenths of an eV) as a function of film thickness, ${ }^{2,16}$ e.g., from $1.23 \mathrm{eV}$ for ML 6T/ $\mathrm{Ag}(111)$ to $1.38 \mathrm{eV}$ for multilayers. ${ }^{14}$ We attribute the origin of the unexpected lower BE of a multilayer DH6T film compared to the ML to the hexyl substitution, and an associated abrupt change in molecular orientation in the thin film after ML completion, as corroborated by in situ AFM studies for DH6T/Ag(111). While the samples with ML coverage exhibited the same morphology as the bare Ag crystal surface, large islands with distinct step heights of about $26 \AA$ were observed for multilayer coverage [Figs. 3(a) and 3(b)]. From this height and the length of a DH6T molecule with the hexyl chains in the all-trans conformation (38.1 $\AA$ ) (Ref. 9) one may estimate a tilt angle of approximately $47^{\circ}$ between the long molecular axis and the surface normal. This angle is larger than the one reported for DH6T on silicon oxide $\left(21.3^{\circ}\right) .^{9}$ However, rodlike molecules are prone to exhibit a number of different crystal structure polymorphs (with dif-
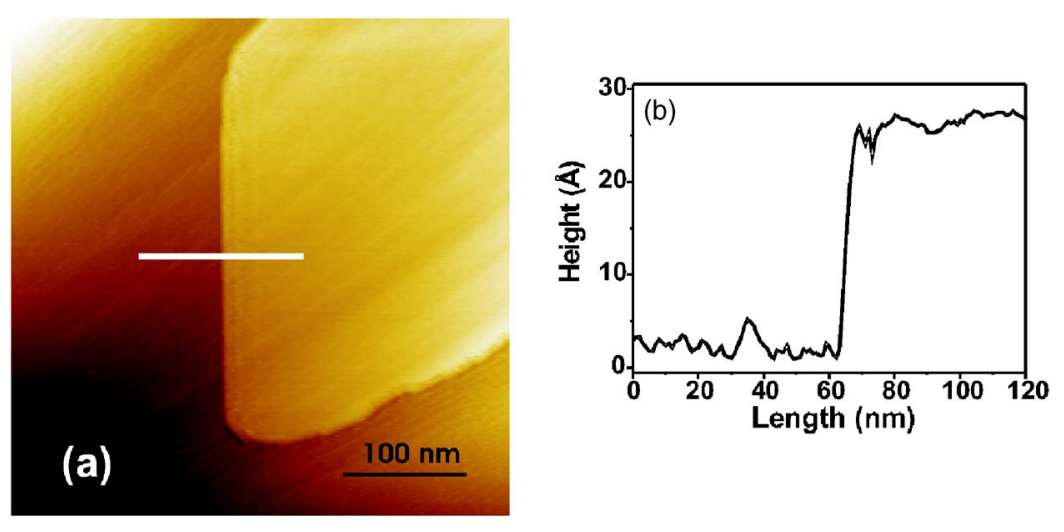

(c)

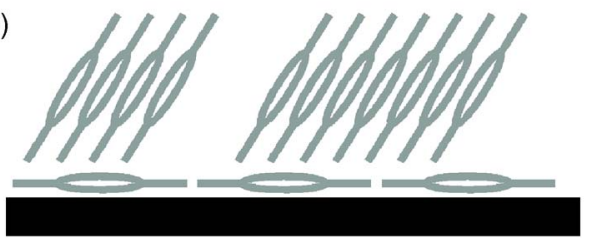

Downloaded 21 Sep 2007 to 131.169.95.147. Redistribution subject to AIP license or copyright, see http://apl.aip.org/apl/copyright.jsp
FIG. 3. (Color online) (a) AFM micrograph of a nominally $20 \AA$ thick DH6T film on $\mathrm{Ag}(111)$ (height scale: $11.8 \mathrm{~nm}$ ). (b) AFM scan height profile along the line indicated in part (a). (c) Sketch of the proposed growth model of DH6T/Ag(111), with lying monolayer and almost standing multilayer. 
ferent inclination angles of the long molecular axis in the unit cell), ${ }^{17}$ whose existence critically depends on the nature of the substrate and deposition conditions. We therefore assume that multilayer DH6T exists in a different polymorph on ML-DH6T/Ag(111) than it does on $\mathrm{SiO}_{2}$; in addition, some alkyl chains may not be in the all-trans conformation. A sketch of the proposed growth model is depicted in Fig. $3(\mathrm{c})$. In fact, a change of the orientation (relative to the substrate surface) of rodlike conjugated organic molecules in thin films as a function of film thickness is a rather common observation, related to the release of stress existing in the ML or few multilayer systems. ${ }^{11,12,15,18}$ However, the changes in orientation are not well understood (in terms of a critical layer thickness that initiates these changes), and substantial changes in BE's derived from photoemission, such as those reported here, were not yet observed.

The defined transition from lying to vertically inclined molecules observed for DH6T/Ag, combined with the insulating properties of the hexyl substituents, leads to fundamentally different electronic properties in comparison to unsubstituted 6T. Apparently, there is a transition from lying to almost standing 6T molecules on metal surfaces as a function of film thickness. ${ }^{11,12}$ However, aside from photoholescreening effects, no significant changes in $\Delta_{h}$ were observed. Since the ML of DH6T is lying flat on the metal surface (see above), the interface energetics are similar to 6T/Ag(111). However, already in the second layer of DH6T the molecules are vertically inclined on the surface, i.e., the conjugated sexithienyl moieties are electronically decoupled from the first ML by insulating hexyl chains. Consequently, a lack of thermodynamic equilibrium may exist between MLDH6T and the multilayer. ${ }^{19}$ Note that the abrupt changes in $\mathrm{BE}$ are not observed for 6T, where sexithienyl moieties are not separated by alkyl chains. The new position of $E_{F}$ within the energy gap of multilayer DH6T may now be reminiscent of the intrinsic Fermi level of the organic material. We note that DH6T grown on Au exhibited qualitatively the same phenomena as reported here for $\operatorname{Ag}(111){ }^{20}$

In conclusion, combined UPS and AFM experiments showed that the growth of DH6T on $\mathrm{Ag}(111)$ exhibits a flat lying first monolayer, followed by a second layer, where the long molecular axis is inclined substantially relative to the surface. This growth results in an electronic decoupling of the second from the first molecular layer, via hexyl chains.
The consequence is a rigid shift of molecular levels in the second layer towards lower binding energy by $0.5 \mathrm{eV}$ compared to the monolayer. The hole injection barrier for the DH6T multilayer on $\mathrm{Ag}(111)(0.60 \mathrm{eV})$ is significantly lower than for the unsubstituted $6 \mathrm{~T}(1.4 \mathrm{eV})$.

The authors thank H.C. Starck GmbH for providing DH6T. One of the authors (N.K.) acknowledges financial support by the Emmy Noether Program (DFG).

${ }^{1}$ M. Fahlmann and W. R. Salaneck, Surf. Sci. 500, 904 (2002).

${ }^{2}$ A. Kahn, N. Koch, and W. Gao, J. Polym. Sci., Part B: Polym. Phys. 41, 2529 (2003)

${ }^{3}$ I. G. Hill, D. Milliron, J. Schwartz, and A. Kahn, Appl. Surf. Sci. 166, 354 (2000).

${ }^{4}$ Conjugated Polymer and Molecular Interfaces: Science and Technology for Photonic and Optoelectronic Applications, edited by W. R. Salaneck, K. Seki, A. Kahn, and J.-J. Pireaux (Dekker, New York, 2001).

${ }^{5}$ Z. L. Li, S. C. Yang, H. F. Meng, Y. S. Chen, Y. Z. Yang, C. H. Liu, S. F. Horng, C. S. Hsu, L. C. Chen, J. P. Hu, and R. H. Lee, Appl. Phys. Lett. 84, 3558 (2004).

${ }^{6}$ H. E. Katz, J. Mater. Chem. 7, 369 (1997).

${ }^{7}$ T. Schwieger, X. Liu, H. Peisert, B. Adolphi, N. Kiriy, and M. Knupfer, J. Appl. Phys. 97, 123712 (2005).

${ }^{8}$ J. Ackermann, C. Videlot, P. Raynal, A. El Kassimi, and P. Dumas, Appl. Surf. Sci. 212-213, 26 (2003).

${ }^{9}$ F. Garnier, A. Yassar, R. Hajlaoui, G. Horowitz, F. Deloffre, B. Servet, S. Ries, and P. Alnot, J. Am. Chem. Soc. 115, 8716 (1993).

${ }^{10}$ R. Friedlein, X. Crispin, C. Suess, M. Pickholz, and W. R. Salaneck, J. Chem. Phys. 121, 2239 (2004).

${ }^{11}$ G. Yoshikawa, M. Kiguchi, S. Ikeda, and K. Saiki, Surf. Sci. 559, 77 (2004).

${ }^{12}$ C. E. Heiner, J. Dreyer, I. V. Hertel, N. Koch, H.-H. Ritze, W. Widdra, and B. Winter, Appl. Phys. Lett. 87, 093501 (2005).

${ }^{13}$ R. L. Johnson and J. Reichardt, Nucl. Instrum. Methods Phys. Res. 208, 719 (1983).

${ }^{14}$ N. Koch, G. Heimel, J. Wu, E. Zojer, R. L. Johnson, J.-L. Bredas, K. Müllen, and J. P. Rabe, Chem. Phys. Lett. 413, 390 (2005).

${ }^{15} \mathrm{G}$. Witte and Ch. Wöll, J. Mater. Res. 19, 1889 (2004).

${ }^{16}$ H. Ishii, K. Sugiyama, E. Ito, and K. Seki, Adv. Mater. (Weinheim, Ger.) 11, 605 (1999).

${ }^{17}$ R. Ruiz, A. C. Mayer, G. G. Malliaras, B. Nickel, G. Scoles, A. Kazimirov, H. Kim, R. L. Headrick, and Z. Islam, Appl. Phys. Lett. 85, 4926 (2004).

${ }^{18}$ G. Beernink, T. Strunskus, G. Witte, and Ch. Wöll, Appl. Phys. Lett. 85, 398 (2004).

${ }^{19}$ N. Koch, C. Chan, A. Kahn, and J. Schwartz, Phys. Rev. B 67, 195330 (2003).

${ }^{20}$ S. Duhm, H. Glowatzki, R. L. Johnson, J. P. Rabe, and N. Koch (unpublished). 